\title{
Favorable clinical course after early- intensive immunotherapy for new-onset refractory status epilepticus
}

\section{CASE REPORT}

Received: December 11, 2019

Revised: January 23, 2020

Accepted: February 6, 2020

Corresponding Author:

Sung-Ho Ahn, MD

Department of Neurology, Pusan

National University Yangsan Hospital,

Pusan National University School of

Medicine, 20 Geumo-ro, Mulgeum-

eup, Yangsan 50612, Korea

Tel: +82-55-360-2122

Fax: +82-55-360-2152

E-mail: caesarahn11@gmail.com

Background: New-onset refractory status epilepticus (NORSE) refers to the newly established concept of a disease characterized by refractory status epilepticus without an identifiable etiology. Recent reports have indicated the importance of immunotherapy for NORSE.

Case Report: A 37-year-old man with no past history of epilepsy was admitted for a presenting complaint of confusion. He was treated with acyclovir and anti-epileptic drug (AED) for presumed herpes encephalitis. However, he developed generalized tonic-clonic seizures on day 6 of admission that worsened despite treatment with multiple AEDs. NORSE was considered to be a probable diagnosis and immunotherapy with methylprednisolone and immunoglobulin was scheduled. However, persistent seizure activity was observed on the electroencephalogram after the completion of initial immunotherapy. Subsequently, rituximab was administered for 4 weeks. He eventually regained consciousness and was able to resume social activity.

Conclusion: Our patient exhibited a favorable outcome with early-intensive immunotherapy and subsequent rituximab treatment for NORSE.

Keywords: New-onset refractory status epilepticus; Immunotherapy; Rituximab

\section{INTRODUCTION}

Although there is lack of consensus regarding the definition of refractory status epilepticus (RSE), it can be defined as continuous or repetitive seizures that do not respond to first and second-line antiepileptic drug (AED) therapy [1]. RSE is considered to be a life-threatening emergency with a high mortality rate of at least $15 \%$ or even $40 \%$, if it is left untreated [2].
Currently, immunotherapy is thought to be an important newly established concept for treating new-onset refractory status epilepticus (NORSE), which is characterized by RSE, without an identifiable etiology in otherwise healthy individuals [3].

We describe a favorable outcome in a patient with NORSE, who was treated with early-intensive immunotherapy in the form of sequential administration of intravenous methylprednisolone and immunoglobulin, followed by subsequent rituximab adminis-

(C) 2020 The Korean Neurocritical Care Society

This is an Open Access article distributed under the terms of the Creative Commons Attribution Non-Commercial License (http://creativecommons.org/licenses/by-nc/4.0/) which permits unrestricted noncommercial use, distribution, and reproduction in any medium, provided the original work is properly cited. 
tration within 2 weeks of initiating immunotherapy.

\section{CASE REPORT}

A 37-year-old man with no past history of epilepsy was transferred to our center. He presented with confusion preceded by fever, chills, and headache. Baseline laboratory tests and computed tomography imaging revealed no definite evidence of systemic inflammation/infection. Cerebrospinal fluid (CSF) examination was unremarkable, except for a mild elevation in CSF proteins $(60.7 \mathrm{mg} / \mathrm{dL})$, with an opening pressure of $16 \mathrm{~cm}$, no cells (0/ $\mu \mathrm{L}$ ), and a glucose level of $95 \mathrm{mg} / \mathrm{dL}$ (for comparison, the serum glucose level was $150 \mathrm{mg} / \mathrm{dL}$ ). Red blood cells were not detected on CSF. Staining, culture, antibodies, and polymerase chain reaction tests for the detection of bacteria, fungi, viruses, and mycobacterium tuberculosis in the CSF were negative. CSF cytology for the detection of abnormal malignant cells was also negative. Serum influenza virus A/B antigen, herpes simplex virus, and varicella-zoster virus antibodies, blood culture, and autoimmune antibodies (e.g., antinuclear, antidouble-stranded DNA, antithyroid peroxidase, antithyroglobulin, and antineutrophilic cytoplasmic antibodies) were absent. Brain magnetic resonance imaging (MRI) revealed a diffusion restricted lesion in the right medial temporal lobe with increased gadolinium enhancement (Fig. 1A, red line). Baseline electroencephalogram (EEG) (obtained at admission) revealed slow background activity.

The patient was administered acyclovir $10 \mathrm{mg} / \mathrm{kg}$, every 8 hours, and levetiracetam $500 \mathrm{mg}$, every 12 hours, following a probable diagnosis of herpes encephalitis. However, episodes of focal seizures with lip smacking and eyeball deviation to the left side increased in frequency, which were accompanied by a newly developed epileptiform discharge on the EEG that required multiple AEDs: valproic acid $600 \mathrm{mg}$, every 8 hours; phenytoin 200 $\mathrm{mg}$, every 8 hours; levetiracetam 1,000 mg, every 12 hours; and phenobarbital $100 \mathrm{mg}$, every 8 hours.

On day 6, he developed a generalized tonic-clonic seizure, which lasted for more than 5 minutes, which worsened, and led to a drastic increase in the need for multiple anesthetics and AEDs. A probable diagnosis of NORSE was made at this stage and immunotherapy was scheduled (Fig. 1B). Intravenous methylprednisolone and immunoglobulin were sequentially administered, which alleviated the seizure activity, thereby reducing the need for anesthetics. However, persistent seizure activity was noted on the EEG 3 days after the completion of intravenous methylprednisolone and immunoglobulin treatment (Fig. 1C). Rituximab was administered, and the seizure activity resolved completely on EEG 4 days after the first infusion, prompting discontinuation of anesthetic infusion. He regained consciousness and could follow instructions 4 days after the discontinuation of anesthetics.

At this stage, the test results for the detection of autoimmune encephalitis antibodies (e.g., NMDAR, AMPA1, AMPA2, LGI1, CASPR2, and GABA-B) and paraneoplastic antibodies (e.g., Hu, Yo, Ri, Ma2, CV2/CRMP5, amphiphysin, recoverin, SOX1, and titin) in serum and CSF were negative. Follow-up brain MRI revealed an improvement in what was probably herpes encephalitis or limbic encephalitis compared to the earlier lesion on the baseline image (Fig. 1A, blue line). He completed a 4-week schedule of rituximab treatment and successfully returned to social activity after 2 months of hospitalization.

\section{DISCUSSION}

We described the favorable outcome of early-intensive immunotherapy for a 37-year-old man with RSE. Treatment was initiated immediately on suspicion of NORSE with a 5-day course of intravenous methylprednisolone and immunoglobulin, followed by the first infusion of rituximab within 2 weeks of immunotherapy. This drug regimen kept the patient seizure-free for a period of 1-month after hospitalization.

Although NORSE was initially defined as cryptogenic RSE, recent studies have suggested that autoimmune encephalitis may be a common etiology $[4,5]$. Therefore, immunotherapy has been recommended for NORSE, even in the absence of the detection of specific antibodies, after excluding infections from the differential diagnosis [6]. Furthermore, several recent systematic reviews have reported that early treatment is associated with improved outcomes in autoimmune encephalitis [5]. Therefore, earlier empirical immunotherapies such as steroid-pulse therapy, immunoglobulins, or plasmapheresis are often tried sequentially, depending on the clinical response.

Interestingly, although our patient exhibited improvement in the clinical course during initial immunotherapy (e.g., sequential treatment with intravenous methylprednisolone and immunoglobulin), which contributed to a dramatic decrease in the need for anesthetics, total discontinuation of anesthetics was possible with the subsequent administration of rituximab (for the management of RSE). Rituximab is a monoclonal antibody against CD20-positive B cells. It leads to B-cell depletion and therefore, suppression of autoimmune neurological disorders including autoimmune encephalitis, especially in patients that respond poorly or are unresponsive to empirical immunotherapy $[7,8]$. Rituximab does not affect the innate immune system, immunoglobulin, or T-cell activity, and is relatively safe in terms of infectious adverse events [9]. Therefore, rituximab can be considered to be an 
A

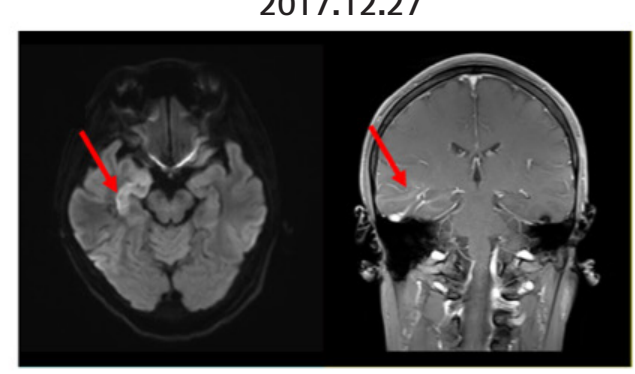

2018.02.21

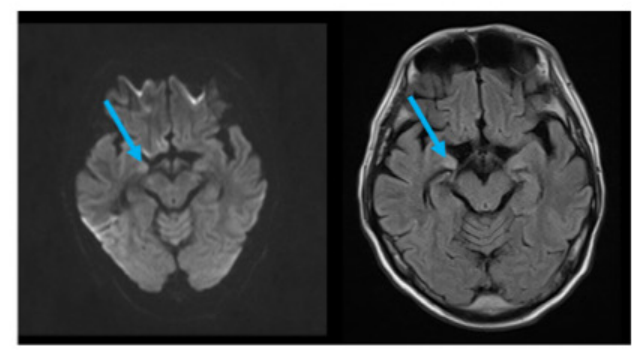

B

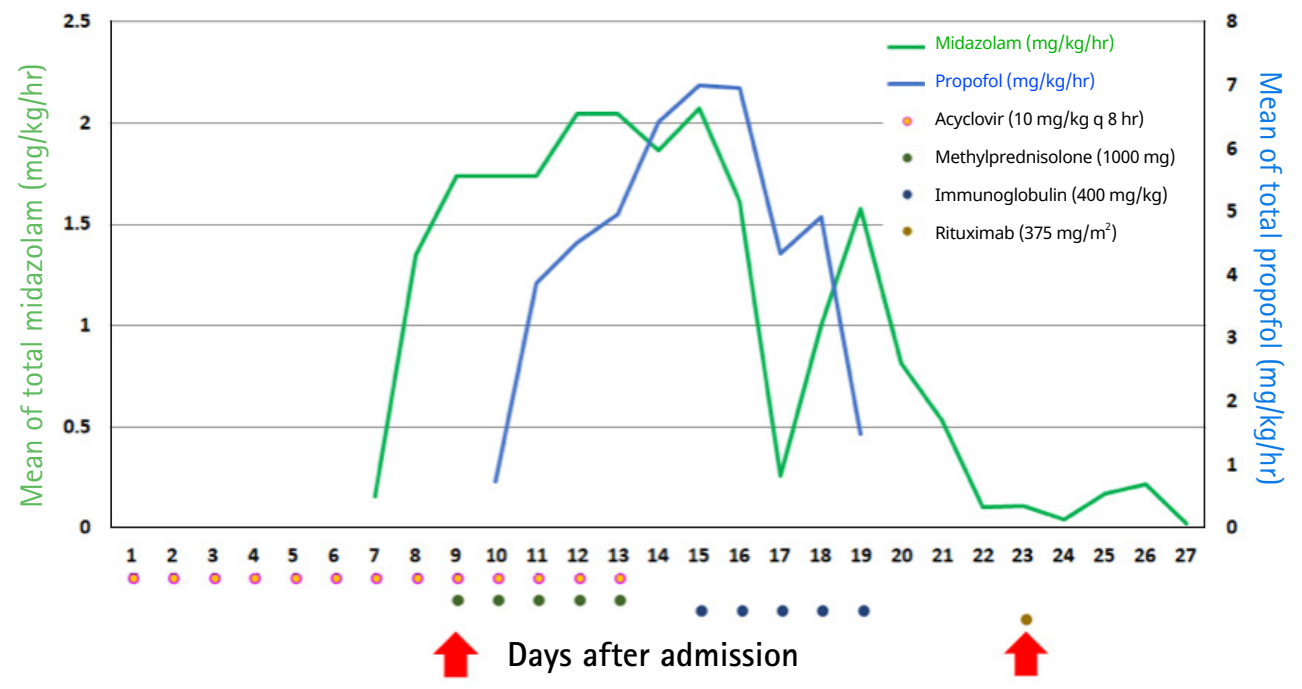

C

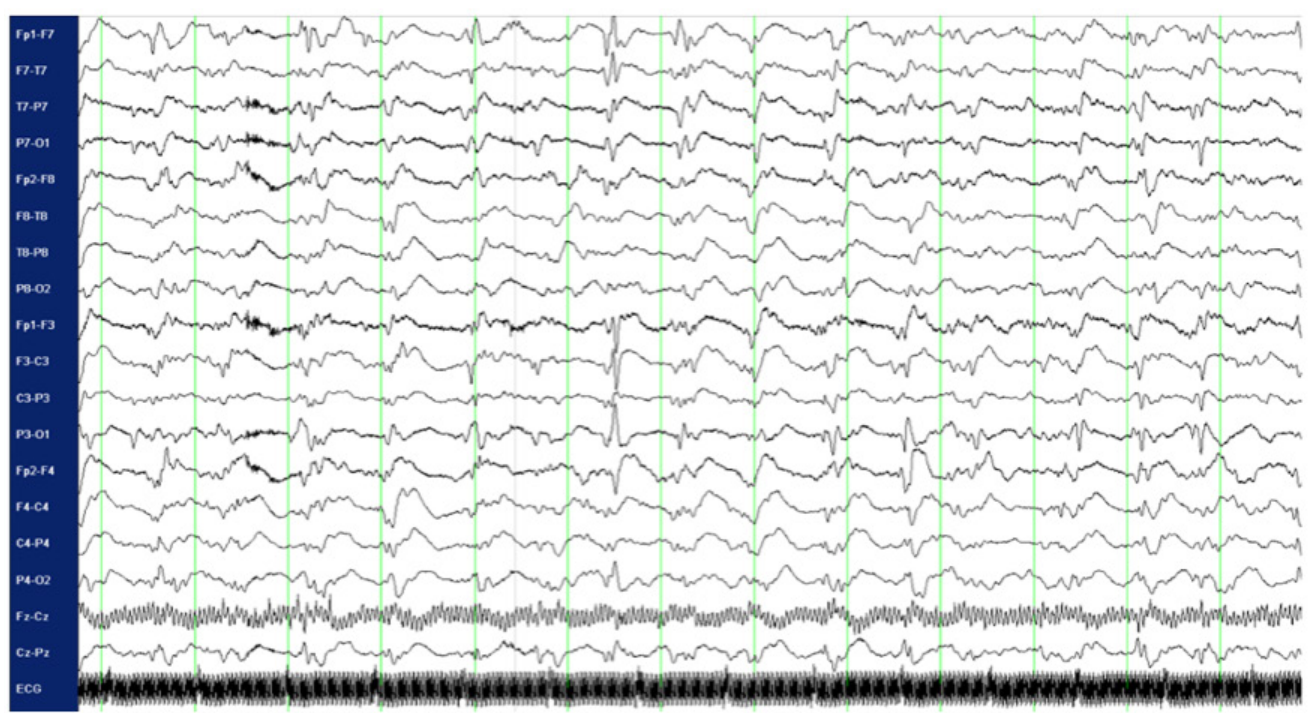

Fig. 1. (A) Follow-up brain magnetic resonance imaging showing disappearance of the diffusion-restricted lesion with remnant fluidattenuated inversion recovery changes in the right medial temporal lobe (blue lines), which previously showed an increased diffusion restriction and gadolinium enhancement (red lines). (B) Continuous infusion of multiple anesthetics including midazolam and propofol was increased sequentially for the management of refractory status epilepticus, but were tapered during sequential immunotherapy (methylprednisolone 1,000 mg for 5 days and intravenous immunoglobulin [IVIG] $0.4 \mathrm{~g} / \mathrm{kg}$ for 5 days), followed by the first infusion of rituximab $375 \mathrm{mg} / \mathrm{m}^{2}$ as booster immunotherapy (red arrow), after the maximum possible administration of multiple antiepileptic drugs including levetiracetam 1,500 mg, every 12 hours; valproic acid 800 mg, every 6 hours; phenytoin 300 mg, every 8 hours; phenobarbital 200 mg, every 8 hours; and topiramate 200 mg, every 12 hours. (C) Rituximab was started on day 23 of hospitalization, based on the findings of bilateral periodic lateralized epileptiform discharge on continuous electroencephalography monitoring even 3 days after the completion of initial immunotherapy, with a regimen of once a week for 4 weeks. 
early immune booster for patients with NORSE, who are resistant to empirical immunotherapy, with or without proven autoantibodies. Prolonged seizure activity can aggravate epileptogenicity, which in turn leads to an increased risk of super-RSE by a kindling mechanism [10].

\section{ARTICLE INFORMATION}

\section{Conflict of interest}

No potential conflict of interest relevant to this article.

\section{Funding}

This work was supported by a 2-year Research Grant of Pusan National University.

\section{ORCID}

Hyun-Sung Kim, https://orcid.org/0000-0002-2634-7569

Jiyoung Kim, https:/ /orcid.org/0000-0001-7592-2921

Bo-Jin Hwang, https: / / orcid.org/0000-0002-9264-8036

Kyoung-Nam Woo, https://orcid.org/0000-0001-8115-0905

Min-Gyu Park, https://orcid.org/0000-0003-2968-6328

Kyung-Pil Park, https://orcid.org/0000-0003-4952-3796

Sung-Ho Ahn, https://orcid.org/0000-0002-8376-545X

\section{Author contributions}

Conceptualization: HSK, SHA. Visualization \& Writing-original draft: HSK, JK, BJH, KNW, MGP, KPP, SHA. Writing-review editing: HSK, JK, BJH, KNW, MGP, KPP, SHA.

\section{REFERENCES}

1. Mayer SA, Claassen J, Lokin J, Mendelsohn F, Dennis LJ, Fitz- simmons BF. Refractory status epilepticus: frequency, risk factors, and impact on outcome. Arch Neurol 2002;59:205-10.

2. Hocker SE, Britton JW, Mandrekar JN, Wijdicks EF, Rabinstein AA. Predictors of outcome in refractory status epilepticus. JAMA Neurol 2013;70:72-7.

3. Gaspard N, Hirsch LJ, Sculier C, Loddenkemper T, van Baalen A, Lancrenon J, et al. New-onset refractory status epilepticus (NORSE) and febrile infection-related epilepsy syndrome (FIRES): state of the art and perspectives. Epilepsia 2018;59: 745-52.

4. Shin JW, Koo YS, Kim YS, Kim DW, Kim KK, Lee SY, et al. Clinical characterization of unknown/cryptogenic status epilepticus suspected as encephalitis: a multicenter cohort study. J Neuroimmunol 2018;315:1-8.

5. Hermetter C, Fazekas F, Hochmeister S. Systematic review: syndromes, early diagnosis, and treatment in autoimmune encephalitis. Front Neurol 2018;9:706.

6. Gaspard N, Foreman BP, Alvarez V, Cabrera Kang C, Probasco JC, Jongeling AC, et al. New-onset refractory status epilepticus: etiology, clinical features, and outcome. Neurology 2015;85: 1604-13.

7. Dalakas MC. B cells as therapeutic targets in autoimmune neurological disorders. Nat Clin Pract Neurol 2008;4:557-67.

8. Lee WJ, Lee ST, Byun JI, Sunwoo JS, Kim TJ, Lim JA, et al. Rituximab treatment for autoimmune limbic encephalitis in an institutional cohort. Neurology 2016;86:1683-91.

9. Whittam DH, Tallantyre EC, Jolles S, Huda S, Moots RJ, Kim $\mathrm{HJ}$, et al. Rituximab in neurological disease: principles, evidence and practice. Pract Neurol 2019;19:5-20.

10. Kirmani BF, Barr D, Robinson DM, Pranske Z, Fonkem E, Benge J, et al. Management of autoimmune status epilepticus. Front Neurol 2018;9:259. 